Consider the category: The effect of spacing depends on individual learning histories

Word Count: 8,522 (text + references) 


\begin{abstract}
The spacing effect refers to increased retention following learning instances that are spaced out in time compared to massed together in time. By one account, the advantages of spaced learning should be independent of tasks particulars and previous learning experiences, as spacing effects have been demonstrated in a variety of tasks across the lifespan. However, by another account, spaced learning should be affected by previous learning because past learning affects the memory and attention processes that form the crux of the spacing effect. The present study investigated whether individuals' learning histories affect the role of spacing in category learning. We examined the effect of spacing on 24 2- to 3.5-year-old children's learning of categories organized by properties that children's previous learning experiences have biased them to attend to (i.e., shape) and properties they are less biased to attend to (i.e., texture, color). Spaced presentations led to significantly better learning of shape categories, but not texture or color categories, compared to massed presentations. Additionally, Generalized Estimating Equations analyses revealed positive relations between the size of children's "shape-side" productive vocabularies and their shape category learning, and between the size of children's "against-the-system" productive vocabularies and their texture category learning. These results suggest that children's attention to and memory for novel object categories are strongly related to their individual word-learning histories. Moreover, children's learned attentional biases affected the types of categories for which spacing facilitated learning. These findings highlight the importance of considering how learners' previous experiences may influence future learning. Keywords: categorization; children; learning history; shape bias; spacing effect
\end{abstract}


Consider the category: The effect of spacing depends on individual learning histories Spacing repetitions of learning instances over time promotes better memory and generalization than massing learning instances together in time (e.g., Cepeda, Pashler, Vul, Wixted, \& Rohrer, 2006; Childers \& Tomasello, 2002; Donovan \& Radosevich, 1999; Ebbinghaus, 1885/1913; Kornell \& Bjork, 2008). This robust finding has been demonstrated in a variety of category-learning tasks across the lifespan (e.g., Kornell \& Bjork, 2008; see Vlach, 2014), suggesting that the advantages of spaced learning schedules are not tied to particular tasks and may be independent of individuals' previous learning experiences. However, the effects of spacing depend heavily on learners' online memory and attention (e.g., Appleton-Knapp, Bjork, \& Wickens, 2005; Bjork \& Bjork, 1992). Memory and attention, in turn, are heavily influenced by biases individuals acquire through past learning experiences (e.g., Castel, McCabe, Roediger, \& Heitman, 2007; Hershler \& Hochstein, 2009). It is unclear, therefore, whether spacing benefits learning of all types of information equally or whether spacing might preferentially facilitate acquisition of information to which learners' prior experiences bias them to attend.

The present study investigates how the memory and attention dynamics associated with the spacing effect are affected by the biases individuals acquire through their previous learning experiences. We do so by comparing the contents of children's early vocabularies (i.e., the first several hundred words a child produces); such contents are indicators of the object properties to which children's experiences have biased them to attend. We examine the effect of spacing on children's learning of two types of novel object categories - categories organized by properties that children's experiences have biased them to attend to (i.e., shape) and properties they are less biased to attend to (i.e., texture, color). 


\section{The Spacing Effect}

The "spacing effect" refers to the robust phenomenon whereby learning is increased via study that is spaced out compared to study that is massed together in time (Cepeda et al., 2006). One common explanation of the spacing effect is that, counterintuitively, spacing promotes learning by allowing time for forgetting during the interval between successive presentations. That is, forgetting an initial instance has been shown repeatedly to increase the potency of encoding subsequent instances (e.g., Bjork \& Allen, 1970; Cuddy \& Jacoby, 1982; see Bjork, 2014). This is theorized to occur because the less accessible an item is in memory due to forgetting, the more the memory of that item is strengthened (learned) when it is successfully retrieved (Bjork \& Bjork, 1992).

\section{The Spacing Effect Depends on Memory and Attention Processes}

Although spacing can enhance learning, the effect of spacing appears highly dependent upon the learner's online memory and attention. For instance, some forgetting during a spacing interval is optimal, but too much forgetting can be detrimental (e.g., Appleton-Knapp et al., 2005); if a learner completely forgets previous category instances during a spacing interval it may be difficult to abstract relevant category features (Gagné, 1950). Importantly, the rate at which forgetting occurs is thought to depend upon the strength of encoding (Bjork, 2014). Features that are less attended to, and therefore not as strongly encoded, may be forgotten more quickly than features that are better attended.

Previous learning experiences affect memory and attention processes. The memory and attention processes that are important for spacing are heavily influenced by individuals' learning histories. Numerous studies find that prior experiences and domain-specific expertise affect both memory and attention across a variety of domains (e.g., Ericsson \& Kintsch, 1995; 
Geoffrey, Brooks, \& Allen, 1989; Lehmann \& Ericsson, 1996; Hatano \& Osawa, 1983). For example, when reading a passage about a baseball game, junior-high students' short- and longterm retention of the passage were dependent upon students' prior knowledge of baseball (Recht \& Leslie, 1988). In another study, individuals studied a word list containing animal names that represent American football teams (e.g., broncos; Castel et al., 2007). Individuals with high levels of knowledge about football correctly recalled more animal names, but also suffered from more intrusions of nonpresented animal names that represented football teams, compared to individuals with low levels of football knowledge. Such findings suggest that an individual's learning history can both facilitate and interfere with future memory and learning.

Research also suggests that prior experience's influence on memory and attention extends to categorization tasks. For instance, adults trained to be experts at identifying 'Greebles'—novel objects that share a common spatial configuration—-showed enhanced memory for specific Greebles, as well as better categorization of novel Greebles several months after training, compared to novices (Gauthier, Williams, Tarr, Tanaka, 1998). In categorization tasks with young children, training with actions that draw attention to different properties of a category exemplar systematically changes the range of test items to which children extend category labels (Smith, 2005). Similarly, word labels that draw attention to different category boundaries affect the category prototypes infants form (Althaus \& Westermann, in press). Together with the aforementioned memory studies, this research demonstrates that past learning experiences can significantly affect memory and attention during future learning and categorization. The present study examines whether such effects of learning history on memory and attention influence whether and how learning benefits from spacing. 


\section{Children's Early Vocabularies and Spaced Learning}

The present study examines one type of learning history, attentional biases reflected in children's early vocabularies, and its impact on the spacing effect in children's category learning. Because the term category often means different things to different people, we first describe how the term is used in this paper: The term category refers to a set of distinct objects to which a common word label is applied. Similar definitions have been widely used in the Psychology literature (e.g., Bruner, Goodnow, \& Austin, 1956; Mervis \& Rosch, 1981; Neisser, 1987). Several recent studies have examined the effect of spacing on children's category learning (e.g., Vlach, Ankowski, \& Sandhofer, 2012; Vlach, Sandhofer, \& Kornell, 2008; Vlach, Sandhofer, \& Bjork, 2014). For instance, Vlach et al. (2008) examined the effect of spacing on 3-year-olds' acquisition of labels for novel object categories organized by similarity in shape. Children were presented with three category exemplars presented either in immediate succession ("massed" condition) or with 30-second play intervals between exemplars ("spaced" condition). Vlach et al. found that children correctly extended category labels to novel objects matching in shape more often following spaced compared to massed presentations. Similarly, Vlach et al. (2012) also found a benefit of spacing on 2-year-olds' categorization of novel objects organized by similarity in shape.

Vlach and colleagues $(2008,2012,2014)$ concluded from such studies that the forgetting induced by spacing promotes category induction. Specifically, they argue that forgetting facilitates categorization by promoting the memory of relevant category features and deterring the memory of irrelevant features. However, the evidence for this argument is based on Englishlearning children's generalization of labels for categories organized by similarity in shape, a feature that English-learning children are typically strongly biased to attend to by around 18-24 
months of age (e.g., Gershkoff-Stowe \& Smith, 2004; Smith, 2000, 2001; see "The Shape Bias and Spaced Learning" below). It is possible that the effect of spacing might be influenced by learned attentional biases, such that spacing might not affect children's categorization of all types of information equally.

\section{The Shape Bias and Spaced Learning}

Children's learning histories may affect the impact of spacing on category learning by differentially promoting learning of shape-based object categories, but not learning of categories organized by other dimensions. Around 18-24 months of age, English-learning children begin to show a bias to attend to object shape and map new words to object shape rather than to texture or color (e.g., Gershkoff-Stowe \& Smith, 2004; Smith, 2000, 2001). This "shape bias" is thought to arise because children's early English vocabularies are dominated by object names that refer to solid things belonging to categories organized by shape (e.g., ball, cup). The shape bias is also observed in some other languages, though not as robustly (e.g., Gathercole \& Min, 1997; Hahn \& Cantrell, 2012; Waxman, Senghas, \& Benveniste, 1997).

For instance, Samuelson and Smith (1999) asked adults to judge whether the 312 nouns on the MacArthur-Bates Communicative Developmental Inventory referred to a category of items (1) characterized by solidity or nonsolidity, (2) organized by similarity in shape or in material, and (3) whether each noun was a mass or count noun. Samuelson and Smith found that young children's productive vocabularies consisted primarily of nouns judged by adults to be count nouns that name solid objects organized by similarity in shape (e.g., ball, cup). These socalled "shape-side" nouns (Perry \& Samuelson, 2011) are thought to train children to attend primarily to objects' shapes, such that with increased shape-side vocabulary, a child's shape bias becomes even more robust (see Smith \& Samuelson, 2006). 
When young children with a shape bias are learning shape-based categories, memory and attention dynamics may facilitate categorization. Specifically, children may attend less to features like texture and color, compared to shape, and therefore forget these features more quickly than shape during a spacing interval. Although this forgetting likely facilitates retrieval and abstraction of shape (Vlach et al., 2008), it may impair learners' ability to acquire categories with central properties other than shape.

Recent research suggests that the extent to which learning benefits from spacing depends upon the degree to which the information to be learned is vulnerable to forgetting. For instance, spaced study of brand advertisements (Appleton-Knapp et al., 2005) resulted in adults' recall of significantly less information when the to-be-learned information was more vulnerable to forgetting (i.e., due to context variation). Thus, in contrast to previous assertions (Vlach et al., 2008, 2012, 2014), we contend that during the developmental period when children are likely to exhibit a shape bias — such that features other than shape are vulnerable to forgetting — spacing may not promote, and may even be detrimental to, children's ability to categorize by non-shape features.

\section{Individual Differences in Children's Word-Learning Histories}

Nevertheless, there are individual differences in children's word-learning histories that might make some children more likely than others to attend to shape versus non-shape features. Perry, Axelsson, and Horst (2015) found that 2-year-olds who produced more shape-side nouns were more accurate at remembering objects' shapes than 2-year-olds who produced fewer shapeside nouns. Additionally, Perry and Saffran (2016) found that toddlers who said more, compared to fewer, shape-side nouns were less affected in recognizing a target object whose color had been 
changed, suggesting that color information was less central to the object representations of children with larger shape-side vocabularies.

Moreover, although children's early vocabularies are dominated by shape-side nouns, children also learn "material-side" nouns — nouns judged by adults to be mass nouns that name non-solids organized by similarity in material (e.g., water, pudding) — and "against-the-system" nouns_-nouns that do not support the link between solidity and attention to shape (e.g., chalk, ice, which are solid but belong to categories organized by material; Perry \& Samuelson, 2011). Knowing more material-side and against-the-system nouns, which label categories organized by properties other than shape, might make children more likely to attend to properties in addition to shape. For instance, Perry and Samuelson (2011) found that the more against-the-system nouns 1.5-year-old children had in their productive vocabularies, the more likely they were to extend novel object labels based on similarity in material.

Thus, children's individual learning histories, reflected in the contents of their productive vocabularies, may predispose some children to attend to shape and/or non-shape features more so than other children. Such differences in attention to specific object features may result in individual differences in the effect of spacing on children's learning of shape and non-shape categories.

\section{The Present Study}

The present study investigated whether individuals' learning histories affect the role of spacing in children's category learning. In contrast to Vlach and colleagues' $(2008,2012,2014)$ contention - that the forgetting induced by spacing facilitates categorization by promoting the memory of relevant category features (emphasis added) — we argue that the forgetting induced by spacing may only facilitate categorization by particular object features, dependent upon the 
past experiences of the learner. We examined the effect of spacing on children's learning of novel shape, texture, and color categories. Spacing may promote categorization based on features to which children have learned to attend (i.e., shape), because such information is likely to be represented strongly enough in memory to be retrieved after a spacing delay. In contrast, spacing may impair children's ability to categorize by features to which they have learned to pay less attention (i.e., texture, color), as such features may be less easily recalled after a spacing delay. As young children's attention to different object features has been shown to differ as a function of productive vocabulary, we also analyzed the contents of children's vocabularies — shape-side, material-side, and against-the-system nouns - and how these contents related to categorization performance.

\section{Method}

\section{Participants}

The participants were 242 - to 3.5-year-old $(M=30.5$ months, $S D=4.7$, range $=22$ to 42 months) monolingual English-speaking children. This relatively large age range was recruited to obtain a sample with a broad range of experiences in learning various types of nouns and a broad range of performances on the categorization task. Half the children were randomly assigned to participate in the massed condition and the other half were assigned to the spaced condition. Power analyses based on the effect size $\left(\eta_{p}{ }^{2}=.69\right)$ for the spacing effect in children's shape categorization reported in Vlach et al. (2008) indicated that a sample size of 12 infants per condition would yield power $=0.99$. There were an equal number of boys and girls in each condition. Age did not differ significantly between conditions $(t(22)=0.01, p=.990, d=0.01)$. Children learned three category types (shape, texture, color), each type on a separate day (within a span of 3-23 days, $M=10.7$ days, $S D=5.8$ ). Category order was counterbalanced across subjects. 
Data from additional participants were excluded from the final sample due to inability to complete all three sessions $(n=9)$, not passing the pre-test $(n=9)$, parents not returning the vocabulary questionnaire $(n=5)$, failure to understand the task $(n=5)$, and experimenter error $(n=1)$. Children were recruited from preschools in the Los Angeles area and were given a small gift (a book) for their participation.

\section{Stimuli}

Children were presented with 12 novel object categories organized by similarity in shape (4 categories), texture (4 categories), and color (4 categories). Each category consisted of three exemplars that shared a central property (shape, texture, or color) and differed on the other dimensions. For instance, exemplars of a texture category had the same surface texture but varied in color and shape (see Figure 1A). Exemplars of each category were given the same novel label (i.e., wug, fep, zav, blicket, binto, gorp, koba, dax, toma, coodle, tez, or biv). Category-label pairings and the order in which exemplars were presented were randomized for each participant.

At test, four objects were presented (see Figure 1C). One object was a novel instance of the target category (e.g., wug, fep). The second and third objects were novel objects that did not match the target property, but did match a category exemplar on one non-target perceptual feature (i.e., for texture categories, the second test object matched the color of one learning exemplar and the third test object matched the shape of a different learning exemplar); we refer to these objects as the shape/texture/color distracters hereafter. The fourth object was a figurine of a familiar object (e.g., a toy cat, a shoe), the familiar distracter, which was equivalent in size to all of the other objects. Familiar objects that children were likely to already have word labels for (i.e., $>75 \%$ of males and females at 24 months of age produce the label, Dale \& Fenson, 1996) were included in the multiple-choice test to help us identify children that may not have 
understood the task; children who selected the familiar distracter on the majority of trials were excluded from our analyses for failure to understand the task.
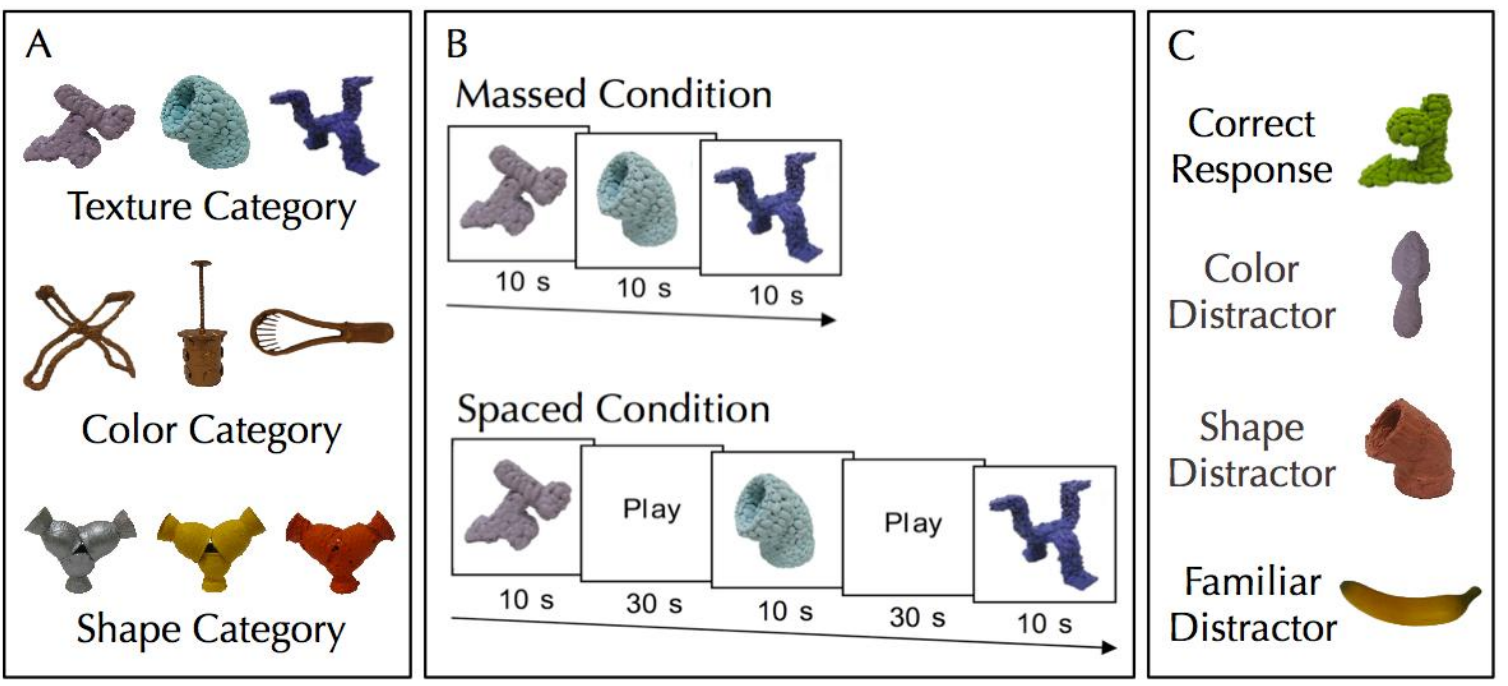

Figure 1. A: Example stimuli sets used in the learning phase of the categorization task. B:

Learning phase procedure shown with texture category stimuli for both presentation timing conditions. C: Example test phase stimuli for texture category.

\section{Design}

The experiment had a 2 (presentation timing) x 3 (category type) design. Presentation timing (massed, spaced) was a between-subjects factor and category type (shape, texture, color) was a within-subjects factor.

\section{Procedure}

Vocabulary assessment. Prior to the first session, parents completed a checklist of 312 early-learned nouns that have been rated for solidity/nonsolidity, mass/count syntax, and organization by similarity in shape versus material (Samuelson \& Smith, 1999). We used this noun checklist to analyze the contents of children's productive vocabularies — shape-side, 
material-side, and against-the-system nouns - and how these contents related to children's learning of the shape, texture, and color categories. Note that we did not include a color vocabulary measure because only three words on the checklist referred to categories organized by similarity in color, but not shape or material (i.e., carrots, pickle, pumpkin; Samuelson \& Smith, 1999).

Comprehension pretest. Children completed a pretest prior to the first session to screen for task comprehension. The comprehension pretest, and all subsequent categorization sessions, were administered individually. A small book and set of keys were labeled one at a time and placed in front of the child. The child was asked to offer the experimenter one object (e.g., "Can you hand me the book?"). After the child made a selection, the objects were presented again and the child was asked to select the other object (e.g., "Can you hand me the keys?"). The experimenter provided neutral feedback (e.g., “Thank you!’) after each selection. Only children who selected the correct object on both trials were asked to participate in the categorization sessions.

Categorization sessions. At the beginning of each categorization session, the child was told that he or she was going to play a game to learn about new toys. Two experimenters conducted each session: One experimenter kept track of the timing and order of presentations, keeping the objects out of the child's sight until presentation. During presentations, the second experimenter kept the object in the child's visual focus at all times, moving the object with the child's gaze if necessary to ensure equivalent looking times across all presentations. During each session, the child was presented with four novel object categories, each consisting of a learning and test phase. 
Learning phase. During the learning phase, three category exemplars were presented either in immediate succession ( $<1 \mathrm{~s}$ between exemplars, massed condition) or with 30 -second play intervals between exemplars (spaced condition; see Figure 1B). Play intervals consisted of reading books, completing puzzles, or playing with play-doh or stickers. In both timing conditions, each exemplar was presented for $10 \mathrm{~s}$ and was labeled three times (e.g., "Look at this wug toy! What a cool wug toy! You hold the wug toy.") An adjectival frame was used to inform the child that an attribute of the object was being labeled, but did not cue attention to any specific property (shape, texture, or color).

Following the learning phase there was a 2-minute retention interval during which children read books, completed puzzles, and/or played with play-doh or stickers. A 2-minute interval was chosen because it (a) required children to access information from long-term memory during the test phase and (b) was short enough to allow children to stay on-task for the entire experiment.

Test phase. Test trials began immediately following the retention interval. The experimenter presented the four test items to children simultaneously saying, "Look at all of these." Children were given up to 30 seconds to explore the test objects. During this time, if a child did not attend to all of the objects, the experimenter touched each object one at a time saying, "Look at this." After the child had attended to each test object, all four objects were gathered by the experimenter, mixed randomly, and pushed toward the child in a horizontal line. The experimenter then asked, "Can you hand me the wug toy?" and held out a hand onto which the child could place an object. Children were given neutral feedback ("Thank you") on their selections. 


\section{Results}

The present study examined whether children's learning histories affect the role of spaced presentation timing in category learning. Specifically, we investigated the possibility that spaced presentation timing may only be beneficial for children's learning of particular types of categories (cf. Vlach et al., 2008, 2012), given the attentional biases engendered by children's previous wordlearning experiences. Although participants' age was not of primary interest, we included age in all of our analyses due to the relatively large age range of our participants.

\section{Does Spacing Promote Learning of Novel Shape, Texture, and Color Categories?}

For each child, we calculated the number of correct responses for each category type (shape, texture, color). We first examined whether the order in which children were taught the different categories affected the number of correct responses for each category type. A 3 (category order: shape vs. texture vs. color learned during the first session) x 3 (category type) repeated measures analysis of variance (ANOVA) did not reveal any significant main effects or interactions $\left(F \mathrm{~s}<1.67, p \mathrm{~s}>.177, \eta_{p}{ }^{2} \mathrm{~s}<.137\right)$. Category order was therefore omitted from further analyses.

Figure 2 shows the average number of correct responses (out of 4) for each category type by children in both presentation timing conditions. As can be seen in the figure, massed presentation timing resulted in near-chance performance ( 1 out of 4 correct) for all category types, whereas spaced presentation timing resulted in different performance for the three category types, suggesting an interaction between presentation timing and category type. A 2 (presentation timing) x 3 (category type) repeated measures ANOVA with age as a covariate, confirmed a significant interaction between presentation timing and category type, $F(1,21)=$ 7.86, $p=.011, \eta_{p}{ }^{2}=.272$. There were no significant main effects or other significant interactions 
$\left(F_{\mathrm{s}}<2.36, p \mathrm{~s}>.139, \eta_{p}^{2} \mathrm{~s}<.102\right)$.

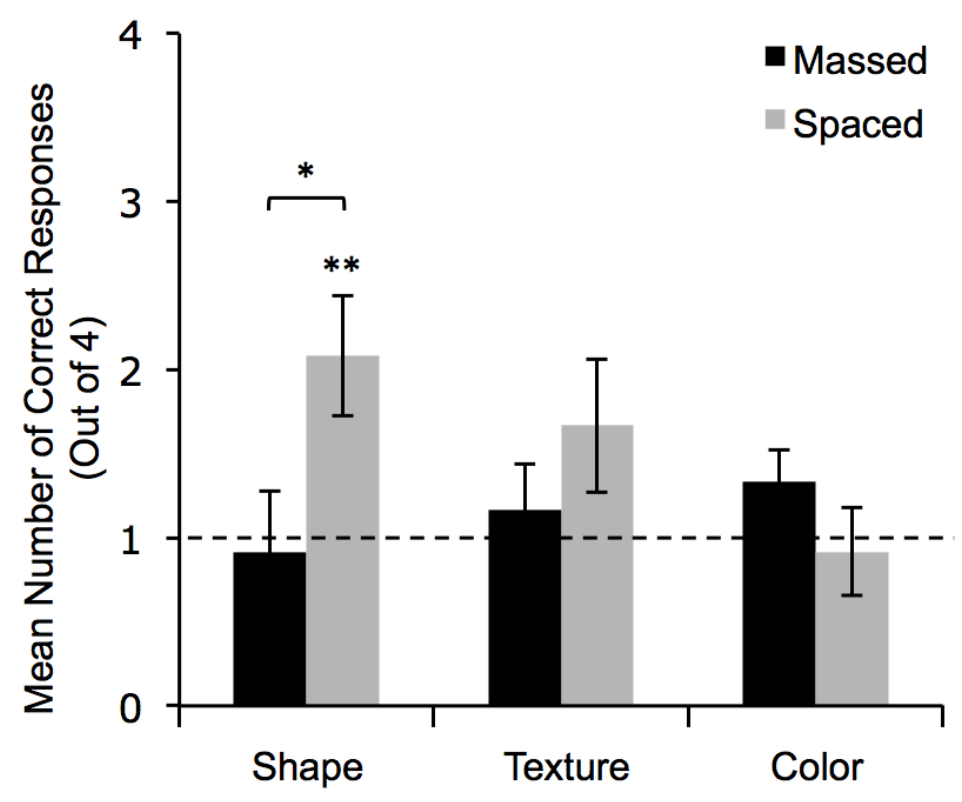

Figure 2. Mean number of correct responses by category type for each of the two presentation timing conditions. Error bars indicate standard errors of the mean. The dashed line represents chance performance (one out of four correct). Asterisks indicate statistical significance between conditions (specified by the bracket) or compared to chance, at the .05 (single asterisk) or .01 (two asterisks) level.

To investigate the interaction between presentation timing and category type, three planned independent samples $t$-tests (this and all future analyses were two-tailed) were conducted. Spaced presentations led to a significantly greater number of correct responses than massed presentations for shape categories $(t(22)=2.30, p=.031, d=0.94)$, but not for texture categories $(t(22)=1.04, p=.308, d=0.43)$, or color categories $(t(22)=1.30, p=.207, d=0.52)$. Moreover, one-sample $t$-tests revealed that only performance on shape categories by children in the spaced presentation timing condition was significantly above chance, $t(11)=3.03, p=.006$, 
$d=0.87$ (all other $t \mathrm{~s}<1.78, p \mathrm{~s}>.103, d \mathrm{~s}<0.51$ ). Thus, we replicated the spacing effect of Vlach and colleagues $(2008,2012)$ with shape, but not texture or color, categories. The effect of spacing on children's categorization performance appears to depend upon the category being learned.

We also examined whether there were systematic patterns to children's errors (shape, color, texture, familiar distracter choices). Different distracters were available for different categories (e.g., the shape distracter was only available as an option for texture and color categories), such that the differences in the number of target choices across categories could bias the number of times particular distracter items were chosen. Thus, rather than analyzing numbers of each type of distracter response, we instead calculated proportions to ask: given that a distracter item was chosen, which was it? For each child, we calculated the proportions of each type of distracter response, out of that child's total number of incorrect responses (see Figure 3). Unlike shape, color, and texture distracters, which were options on $67 \%$ of trials, the familiar distracter was an option on $100 \%$ of trials; therefore, we analyzed familiar distracter responses in a separate analysis from the other distracter responses.

A univariate ANOVA on children's familiar distracter responses yielded no significant effects of age or presentation timing $\left(F_{\mathrm{S}}<1.64, p \mathrm{~s}>.214, \eta_{p}{ }^{2} \mathrm{~s}<.073\right)$. As can be seen in Figure 3 , children chose the familiar distracter less often than predicted by chance $(t(23)=3.57, p=$ $.002, d=0.73$ ). A 2 (presentation timing) x 3 (novel distracter type: shape, color, texture) repeated measures ANOVA with age as a covariate yielded no significant main effects or interactions $\left(F_{\mathrm{S}}<2.00, p \mathrm{~s}>.148, \eta_{p}{ }^{2} \mathrm{~s}<.087\right)$, suggesting that novel distracter responses did not differ significantly by presentation timing, distracter type, or age. As can be seen in Figure 3, children did not chose shape, color, or texture distracters significantly more often than predicted 
by chance $(t \mathrm{~s}<1.52, p \mathrm{~s}>.142, d \mathrm{~s}<0.31)$. Thus, although children were more likely to select a novel distracter compared to a familiar distracter on trials when they did not choose the target, there were no discernable patterns as to which novel distracter children chose.

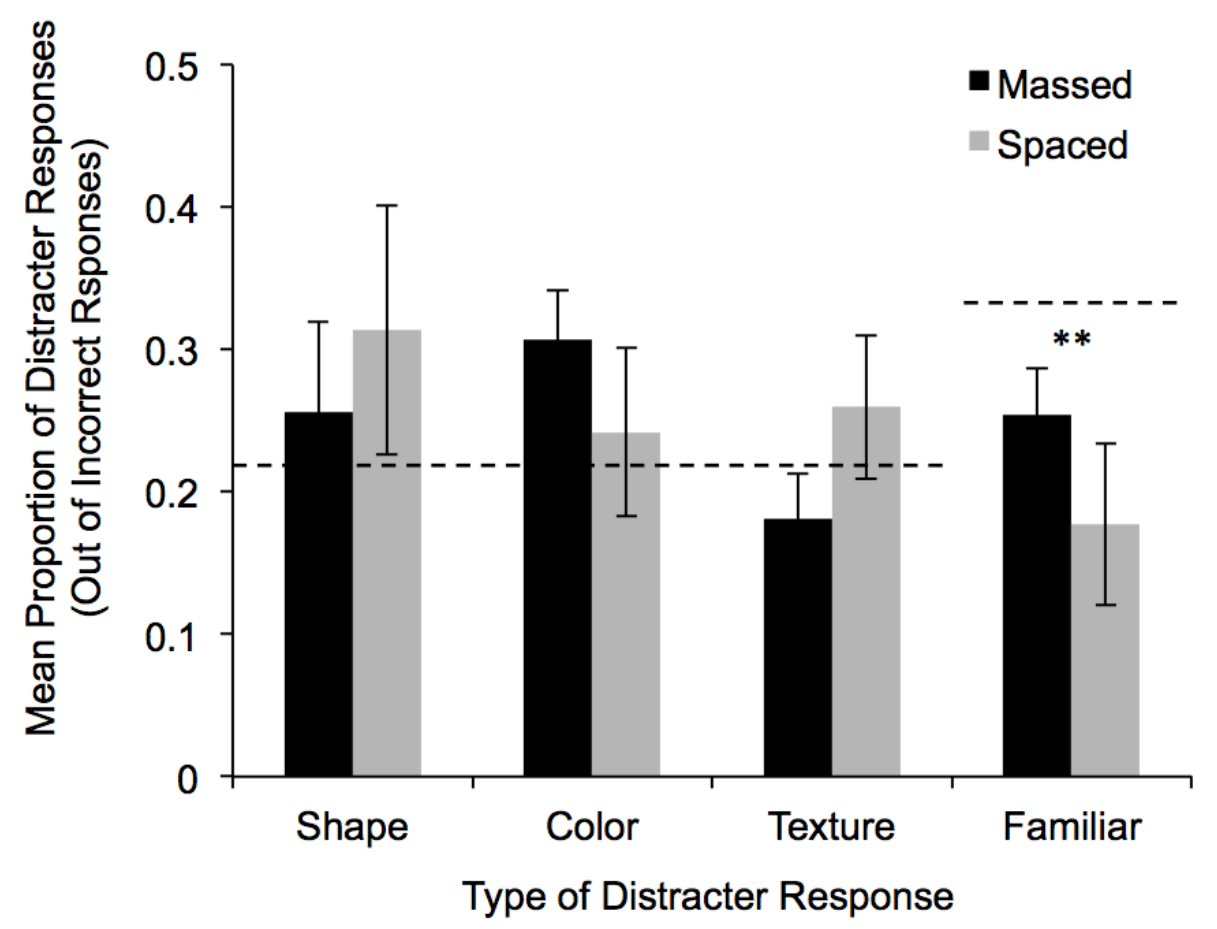

Figure 3. Mean proportion of shape, color, texture, and familiar distracter responses out of all incorrect responses for each of the two presentation timing conditions. Error bars indicate standard errors of the mean. The dashed lines represent chance performance (.22 for shape, color, and texture distracter responses; .33 for familiar distracter responses). Asterisks indicate performance (regardless of presentation timing condition, as described in the text) that is statistically below chance at the .01 level.

\section{Previous Word-Learning Experiences and Categorization Performance}

To investigate why the effect of spacing varied by category type, we analyzed the contents of participants' vocabularies, which are markers of the object features to which children's early 
word-learning experiences have biased them to attend. First, we calculated the number of earlylearned nouns that parents indicated were in each child's productive vocabulary. Previous studies suggest that children with more than 50 nouns in their productive vocabularies are likely to exhibit a shape bias (Gershkoff-Stowe \& Smith, 2004; Samuelson \& Smith, 2009). The children in the present study produced between 128 and $311\left(M_{\text {massed }}=239, S D_{\text {massed }}=50, M_{\text {spaced }}=239, S D_{\text {spaced }}=\right.$ 52) of the 312 nouns on the checklist, suggesting that this population would be highly likely to exhibit a shape bias.

Next, we analyzed the particular types of nouns that composed children's productive vocabularies. Children produced between 73 and 186 shape-side nouns $\left(M_{\text {massed }}=146, S D_{\text {massed }}=\right.$ $\left.28, M_{\text {spaced }}=146, S D_{\text {spaced }}=31\right)$, between 8 and 24 material-side nouns $\left(M_{\text {massed }}=17, S D_{\text {massed }}=6\right.$, $\left.M_{\text {spaced }}=17, S D_{\text {spaced }}=4\right)$, and between 13 and 28 against-the-system nouns $\left(M_{\text {massed }}=22, S D_{\text {massed }}\right.$ $\left.=5, M_{\text {spaced }}=22, S D_{\text {spaced }}=4\right)$. To investigate whether word-learning histories played a significant role in children's category learning, we examined the relation between children's productive vocabulary contents - the number of shape-side, material-side, and against-the-system nouns they produced — and their shape, texture, and color categorization performances. We were also interested in the relative contributions of vocabulary contents and presentation timing to categorization performances, as well as any interactions between vocabulary structure and presentation timing.

Statistical Approach. To estimate these contributions and assess possible interactions, we first needed to identify the correct statistical model. The present count data violate several key assumptions of traditional statistical approaches like ordinary least squares (OLS) regression. For instance, OLS regression assumes that the outcome is a normally distributed continuous variable and that all observations are independent of one another (Berry, 1993). In contrast, the 
outcome variable in the present data - the number of correct responses for each category typetook on a limited range of values and tended to be right skewed. Thus, we treated the response on individual trials as binary (i.e., correctly chose the target, did not choose the target) such that each participant contributed four trials of data for each category type. Because these data fail the assumption of independence of observations, we utilized a regression-based model able to handle both correlated data as well as a variety of outcome variable distributions: Generalized Estimating Equations (GEE; Liang \& Zeger, 1986; Zeger \& Liang, 1986).

Model specification. GEE models require several factors to be specified (Ballinger, 2004). For our binary outcome, we specified a binomial distribution and a logit link function. We chose an exchangeable correlation structure, which assumes that all four trials of each category are equally correlated with each other, for each participant (though GEE models are robust to misspecification of the correlation structure, Zeger \& Liang, 1986).

Model selection. Predictors of shape, texture, and color performances were investigated in separate GEE models. Vocabulary measures were entered into separate models due to multicollinearity among these predictors (see Table 1). Model selection was carried out using the quasi-likelihood-under-the-independence-model-information-criterion (QIC; Pan, 2001). In this model selection process, the model with the lowest QIC score is judged to be the best fit, although absolute QIC score may not be meaningful.

We began by examining all possible main effects models. Each model involved prediction of binary shape, texture, or color performances from a dummy-coded variable comparing the two levels of the presentation timing variable (massed, spaced), from continuous vocabulary variables (total nouns, shape-side nouns, material-side nouns, against-the-system nouns), and/or from age. Once we found the best fitting main effects model, for models 
Table 1

Correlations amongst vocabulary measures and presentation timing

\begin{tabular}{lcccc}
\hline Measure & 1 & 2 & 3 & 4 \\
\hline $\begin{array}{l}\text { 1. Shape-side nouns } \\
\text { 2. Against-the-system nouns }\end{array}$ & $.88^{* *}$ & - & & \\
3. Material-side nouns & $.77 * *$ & $.89 * *$ & - & \\
4. Presentation timing & .02 & -.07 & .04 & - \\
\hline$* * p<.01$. & & & & \\
\hline
\end{tabular}

with more than one predictor we added an interaction term to the model and assessed its significance as well as its affect on the overall model fit. In all cases, models with an interaction term had larger QIC values (worse fit), and non-significant interaction coefficients. Table 2 presents five models of children's shape, texture, and color performances. Although only one of the models provides the best fit for a particular category-Model 1 for shape, Model 2 for texture, and Model 5 for color - the results of five models are shown for all three category types for purposes of comparison. For each model, coefficient estimates (Bs), standard errors (SEs), odds ratios (OR), and 95\% confidence intervals (CIs) are provided for each predictor. We used Wald's chi-square test to assess the statistical significance of individual predictors.

Model interpretation. GEE models produce estimates of the population-averaged effect of each predictor on the outcome, after adjusting for covariates and taking into account withinsubject correlation. For interpretation, ORs are used rather than raw coefficients because the interpretation can be a more intuitive measure of likelihood. ORs that are greater than 1 are interpreted as increasing the likelihood of an outcome whereas ratios less than 1 are interpreted 
as decreasing the likelihood of an outcome. ORs that are equal to 1 are not significantly associated with either increased or decreased risk.

Shape category performance. A model with presentation timing and shape-side noun vocabulary as predictors provided the best fit (lowest QIC) to the shape categorization data (see Table 2, Model 1). Both presentation timing and shape-side noun vocabulary were significant predictors of children's categorization by shape (Wald $\chi^{2} s>4.98, p s<.026$ ). The OR for presentation timing in Model 1 is 3.89, with a 95\% confidence interval of 1.18 to 12.83 . Thus, we can be $95 \%$ confident that the odds of choosing the target on shape category trials were increased by between $18 \%$ and $1183 \%$ for the average participant in the spaced condition compared to the average participant in the massed condition, holding shape-side vocabulary constant. The OR for shape-side vocabulary in Model 1 is 1.02, with a 95\% confidence interval of 1.01 to 1.04 . Thus, we can be $95 \%$ confident that the odds of choosing the target on shape category trials increased by between $1 \%$ and $4 \%$ (on average) for every 1-unit increase in shapeside vocabulary, adjusting for presentation timing condition. This is equivalent to saying that for every 10 -unit increase in shape-side vocabulary, we can be $95 \%$ confident that the odds of choosing the target on shape category trials increased by between $10 \%$ and $48 \%$, adjusting for presentation timing condition.

These findings are consistent with our previous finding that spaced presentation timing facilitated categorization by shape. Moreover, presentation timing is a significant predictor of shape categorization even when controlling for children's shape-side noun vocabulary. These findings also support the importance of the size of children's shape-side noun vocabularies on children's shape category learning. Specifically, although as children produce more words their vocabularies naturally tend to take on the structure of the noun checklist (i.e., mostly shape-side 
Table 2

Model goodness of fit (QIC), coefficient estimates (B), and odds ratios (OR) from five logistic GEE models relating presentation timing and vocabulary measures to children's shape, color, and texture category performances.

\begin{tabular}{|c|c|c|c|c|c|c|c|c|c|c|}
\hline \multirow[b]{2}{*}{ Model } & \multirow[b]{2}{*}{ Independent variable } & \multicolumn{3}{|c|}{ Shape } & \multicolumn{3}{|c|}{ Texture } & \multicolumn{3}{|c|}{ Color } \\
\hline & & $Q I C$ & $B(S E)$ & OR $(95 \% \mathrm{CI})$ & $Q I C$ & $B(S E)$ & OR $(95 \% \mathrm{CI})$ & $Q I C$ & $B(S E)$ & OR $(95 \% \mathrm{CI})$ \\
\hline \multirow[t]{2}{*}{1} & Presentation timing & 118.74 & $1.36 *(.61)$ & $3.89(1.18,12.83)$ & 128.11 & $0.55(.49)$ & $1.73(0.66,4.52)$ & 116.84 & $-0.52(.40)$ & $0.59(0.27,1.31)$ \\
\hline & Shape-side nouns & & $0.02 *(.01)$ & $1.02(1.01,1.04)$ & & $0.01(.01)$ & $1.01(0.99,1.03)$ & & $0.00(.01)$ & $1.00(0.99,1.02)$ \\
\hline 2 & $\begin{array}{l}\text { Against-the-system } \\
\text { nouns }\end{array}$ & 130.33 & $0.10(.07)$ & $1.10(0.97,1.30)$ & 124.64 & $0.12(.07)$ & $1.13(0.99,1.29)$ & 117.15 & $-0.01(.05)$ & $0.99(0.91,1.09)$ \\
\hline 3 & Material-side nouns & 130.50 & $0.08(.06)$ & $1.09(0.97,1.21)$ & 128.43 & $0.06(.06)$ & $1.07(0.96,1.19)$ & 116.69 & $0.00(.04)$ & $1.00(0.93,1.07)$ \\
\hline 4 & Shape-side nouns & 126.08 & $0.02 *(.01)$ & $1.02(1.00,1.04)$ & 127.69 & $0.01(.01)$ & $1.01(0.99,1.03)$ & 117.05 & $0.00(.01)$ & $1.00(0.99,1.02)$ \\
\hline 5 & Presentation timing & 124.95 & $1.30 *(.59)$ & $3.66(1.14,11.72)$ & 128.64 & $0.55(.50)$ & $1.73(0.65,4.62)$ & 115.76 & $-0.52(.41)$ & $0.59(0.27,1.32)$ \\
\hline
\end{tabular}


nouns), shape-side noun vocabulary size was a better predictor of shape category learning than was total noun vocabulary. This finding suggests that what may matter most for learning shape categories is not the total number of nouns a child knows, but the number of them that fall into the "shape-side" classification.

Texture category performance. A model with against-the-system nouns as the only predictor provided the best fit to the texture categorization data (see Table 2, Model 2). Nevertheless, against-the-system vocabulary accounted for only a marginal amount of the variance in texture categorization performances (Wald $\chi^{2}=3.50, p=.061$ ). The OR for againstthe-system nouns in Model 2 is 1.13, with a 95\% confidence interval of 0.99 to 1.29 . Thus, we can be $95 \%$ confident that the odds of choosing the target on texture category trials increased by between $-1 \%$ and $29 \%$ (on average) for every 1 -unit increase in against-the-system vocabulary. These findings suggest that knowing more words that go against the typical classification system of solid objects belonging to categories organized by shape may help children attend to and categorize solid objects based on their textures.

Models 3 and 4 of texture performance, with only a shape-side vocabulary predictor or only a material-side vocabulary predictor, are included in Table 2 for comparison purposes. As can be seen from comparing the results of Models 2-4, although shape-side, material-side, and against-the-system vocabulary sizes were highly correlated (see Table 1) these vocabulary measures appear to account for different amounts of variance in children's categorization performances.

Color category performance. A model with only presentation timing provided the best fit to the color categorization data (see Table 2, Model 5). Nevertheless, presentation timing did not account for a significant amount of the variance in color categorization performances $\left(\right.$ Wald $\chi^{2}=$ 
$1.64, p=.201)$. This finding is consistent with our previous finding that spaced presentation timing does not have a significant effect on color categorization. Moreover, in contrast to our findings with shape and texture categories, the types of nouns children produced did not affect children's color category learning.

\section{Discussion}

Spaced presentations of category exemplars facilitated children's acquisition of shape categories, but showed no benefit compared to massed presentations for texture and color categories. These results are consistent with previous research demonstrating that spacing may promote children's categorization by shape (Vlach et al., 2008, 2012); however the current results also demonstrate that spacing does not benefit all types of category learning equally.

\section{Why did Spacing Only Benefit Shape Category Learning?}

One explanation comes from research demonstrating that forgetting and retrieval difficulty may promote children's categorization based on shape (e.g., Vlach et al., 2008, 2012, 2014). According to such an account, spacing allows for forgetting of object features. Learners must therefore engage in more difficult retrieval to access those features during subsequent exemplar presentations when presentations are spaced compared to when presentations are massed (Vlach et al., 2012). When features from prior presentations are successfully retrieved, memory for those features is strengthened and those features are subsequently forgotten more slowly. However, features that are not successfully retrieved continue to be forgotten and at a faster rate than features that were retrieved (Bjork \& Bjork, 1992; see Bjork, 2014). In sum, spacing promotes forgetting, and therefore more difficult retrieval, than does massing, resulting in stronger memory for information that is successfully retrieved.

Following from such an account, spacing may preferentially benefit children's shape 
category learning because children's attentional biases influence memory for object features. Children in the present study had productive vocabularies dominated by object names that refer to solid objects belonging to categories organized by shape, biasing children's attention to objects' shapes. A shape bias may facilitate the retrieval of shape information following a spacing interval, facilitating abstraction and categorization by shape. Less attention to features like texture and color, in contrast, may make this information less likely to be retrieved after a spacing interval, hindering abstraction and categorization by such features.

This forgetting account of the data is also consistent with consolidation explanations of the spacing effect (e.g., Landauer, 1969). Consolidation accounts explain the spacing effect as a product of memory consolidation during the intervals between learning events. In the present study, spaced presentation timing resulted in longer intervals between category exemplars and therefore a longer learning phase, compared to massed presentation timing, allowing for more memory consolidation prior to test. If a shape bias led children to encode objects' shapes more so than objects' textures or colors, consolidation during learning might preferentially strengthen memory for shape, resulting in a spacing effect primarily for shape categories. Nevertheless, a recent study by Vlach et al. (2014) found differences in 3-year-olds' shape category learning following two different spacing schedules that were equated in time for consolidation during the learning phase. This finding suggests that consolidation may not be a necessary factor underlying the spacing effect in children's shape category learning.

It is also possible that factors other than memory contributed to children's lack of texture and color category learning in the present study. For instance, research suggests that color categories may be particularly difficult dimensional categories for children to learn (Mervis, Bertrand, \& Pani, 1995; Shatz, Behrend, Gelman, \& Ebeling, 1996). Although children acquire 
color categories by 3 to 4 years of age, they nevertheless struggle to learn them (e.g., Backscheider \& Shatz, 1993; Sandhofer \& Smith, 1999). It is possible, if not likely, that factors in addition to memory make color learning difficult, such that even with optimal presentation timing, young children may struggle to acquire color categories.

\section{Vocabulary Structure and Category Learning}

We also analyzed the contents of children's productive vocabularies - shape-side, materialside, and against-the-system nouns - and how these contents related to categorization performance. The size of children's shape-side vocabulary was a significant predictor of shape category performance. This finding suggests that although the children in this study all possessed sizeable shape-side vocabularies (between 73 and 186 shape-side nouns), each additional shape-side noun that a child learned to produce within this range nevertheless resulted in a small but significantly increased likelihood of learning categories organized by shape. Future research could examine whether, in a population with less extensive shape-side vocabularies, knowing more shape-side nouns would result in even larger increases in shape category performance than those observed here.

In contrast to shape categorization, texture categorization was marginally related to the size of children's against-the-system vocabulary. That is, each additional against-the-system noun that a child had learned to produce resulted in a marginally greater likelihood of learning categories organized by texture. The majority of against-the-system nouns in children's productive vocabularies refer to solid objects categorized by material. Knowing more of these nouns may therefore have helped children attend to and categorize the solid objects in the present study based on their textures.

We did not, however, find a significant relation between texture category performance 
and material-side vocabulary. Material-side nouns refer to nonsolid objects categorized by material. Possessing a sizeable material-side vocabulary may not have influenced children's performance because the present task involved categorization of solid objects. Future research could examine whether, in a task involving categorization of nonsolids, having a larger materialside vocabulary would relate to categorization performance.

In contrast to texture category performance, knowing more against-the-system nouns did not help children attend to and categorize objects based on their colors. Of the 28 against-thesystem nouns that children may have known, 25 of them refer to categories of objects organized by similarity in material, whereas only one of them (i.e., green beans) refers to a category organized by similarity in both color and material (Samuelson \& Smith, 1999). Thus, knowing more against-the-system nouns may train children to better attend to the materials, but not the colors, of solid objects. Thus, a vocabulary structure that directs attention away from shape will not necessarily facilitate categorization by non-shape features. Rather, vocabulary structure must also direct attention toward a particular feature to facilitate categorization by that feature.

Interestingly, despite the relatively large age range of the participants in this study, we found no evidence to suggest that increased age resulted in better novel category learning. Rather, GEE analyses suggested that vocabulary composition and/or presentation timing were the strongest predictors of novel category learning. It is possible that with a different age range, perhaps one that included even younger children who may not have yet developed a shape bias, we may have found an effect of age on categorization performance. Nevertheless, the present results suggest that, during the developmental period when children are likely to exhibit a shape bias, children's ability to categorize novel objects by shape and non-shape features is more 
closely linked with children's individual learning histories, as reflected in the contents of their productive vocabularies, than it is with chronological age.

\section{Conclusion}

The present results suggest that children's attention to and memory for novel object categories is strongly related to their individual word-learning histories. Because the effects of presentation timing are influenced by learners' online memory and attention, children's learning histories affected the types of categories for which spacing facilitated learning. These findings highlight the importance of considering how learners' previous experiences may influence future learning.

The present study focused on the shape bias, a particularly prominent attentional bias learned by young children. However, older populations also exhibit attentional biases that affect perception and memory for objects and events (e.g., Drew, Võ, \& Wolfe, 2013; Eitam, Yeshurun, \& Hassan, 2013; Mack \& Rock, 1998). Future research should examine the extent to which these other types of biases and previous learning experiences may affect the memory and attention foundations of the spacing effect. 


\section{References}

Althaus, N., \& Westermann, G. (in press). Labels constructively shape object categories in 10month-old infants. Journal of Experimental Child Psychology, 151, 5-17. http://dx.doi.org/10.1016/j.jecp.2015.11.013

Appleton-Knapp, S. L., Bjork, R. A., \& Wickens, T. D. (2005). Examining the spacing effect in advertising: Encoding variability, retrieval processes, and their interaction. Journal of Consumer Research, 32, 266-276. http://dx.doi.org/10.1086/432236

Backscheider, A. G. \& Shatz, M. (1993). Children's acquisition of the lexical domain of color. In K. Beals et al. (Eds.), What we think, what we mean, and how we say it (Papers from the parasession on the correspondence of conceptual, semantic, and grammatical representation, CLS 29), (Vol. 2, pp. 11-21). Chicago: The Chicago Linguistic Society.

Ballinger, G. A. (2004). Using generalized estimating equations for longitudinal data analysis. Organizational Research Methods, 7, 127-150. http://dx.doi.org/10.1177/1094428104263672

Berry, W. D. (1993). Understanding regression assumptions. Series: Quantitative applications in the social sciences (Vol. 92). Newbury Park, CA: Sage Publications.

Bjork, R. A. (2014). Forgetting as a friend of learning. In D. S. Lindsay, C. M. Kelley, A. P. Yonelinas, \& H. L. Roediger, III (Eds.), Remembering: Attributions, processes, and control in human memory: Papers in honour of Larry L. Jacoby (pp. 15-28). New York: Psychology Press.

Bjork, R. A., \& Allen, T. W. (1970). The spacing effect: Consolidation or differential encoding. Journal of Verbal Learning \& Verbal Behavior, 9, 567-572. http://dx.doi.org/10.1016/S0022-5371(70)80103-7 
Bjork, R. A., \& Bjork, E. L. (1992). A new theory of disuse and an old theory of stimulus fluctuation. In A. Healy, S. Kosslyn, \& R. Shiffrin (Eds.), From learning processes to cognitive processes: Essays in honor of William K. Estes (Vol. 2, pp. 35-67). Hillsdale, NJ: Erlbaum.

Castel, A. D., McCabe, D. P., Roediger, H. L., \& Heitman, J. L. (2007). The dark side of expertise domain-specific memory errors. Psychological Science, 18(1), 3-5. http://dx.doi.org/10.1111/j.1467-9280.2007.01838.x

Cepeda, N. J., Pashler, H., Vul, E., Wixted, J. T., \& Rohrer, D. (2006). Distributed practice in verbal recall tasks: A review ad quantitative synthesis. Psychological Bulletin, 132, 354380. http://dx.doi.org/10.1037/0033-2909.132.3.354

Childers, J. B., \& Tomasello, M. (2002). Two-year-olds learn novel nouns, verbs, and conventional actions from massed or distributed exposures. Developmental Psychology, 38, 967-978. http://dx.doi.org/10.1037/0012-1649.38.6.967

Cuddy, L. J., \& Jacoby, L. L. (1982). When forgetting helps memory: An analysis of repetition effects. Journal of Verbal Learning \& Verbal Behavior, 21, 451-467. http://dx.doi.org/10.1016/S0022-5371(82)90727-7

Dale, P. S., \& Fenson, L. (1996). Lexical development norms for young children. Behavioral Research Methods, Instruments, \& Computers, 28, 125-127. http://dx.doi.org/10.3758/BF03203646

Donovan, J. J., \& Radosevich, D. J. (1999). A meta-analytic review of the distribution of practice effect. Journal of Applied Psychology, 84, 795-805. http://dx.doi.org/10.1037/00219010.84 .5 .795 
Drew, T., Võ, M. L. H., \& Wolfe, J. M. (2013). The invisible gorilla strikes again sustained inattentional blindness in expert observers. Psychological Science, 24(9), 1848-1853. http://dx.doi.org/10.1177/0956797613479386

Eitam, B., Yeshurun, Y., \& Hassan, K. (2013). Blinded by irrelevance: Pure irrelevance induced “blindness". Journal of Experimental Psychology: Human Perception and Performance, 39, 611-615. http://dx.doi.org/10.1037/a0032269

Ebbinghaus, H. (1913). Memory (H. A. Ruger \& C. E. Bussenius, Trans.). New York: Teachers College. (Original work published 1885).

Ericsson, K. A., \& Kintsch, W. (1995). Long-term working memory. Psychological Review, 102(2), 211-245. http://dx.doi.org/10.1037/0033-295X.102.2.211

Gagné, R. M. (1950). The effect of sequence of presentation of similar items on the learning of paired associates. Journal of Experimental Psychology, 40, 61-73. http://dx.doi.org/10.1037/h0060804

Gathercole, V. C. M., \& Min, H. (1997). Word meaning biases or language-specific effects? Evidence from English, Spanish and Korean. First Language, 17(51), 31-56. http://dx.doi.org/10.1177/014272379701705102

Gauthier, I., Williams, P., Tarr, M. J., \& Tanaka, J. (1998). Training 'greeble' experts: A framework for studying expert object recognition processes. Vision Research, 38, 24012428. http://dx.doi.org/10.1016/S0042-6989(97)00442-2

Geoffrey, N. R., Brooks, L. R., \& Allen, S. W. (1989). Recall by expert medical practitioners and novices as a record of processing attention. Journal of Experimental Psychology: Learning, Memory, and Cognition, 15, 1166-1174. http://dx.doi.org/10.1037/02787393.15.6.1166 
Gershkoff-Stowe, L. \& Smith, L. B. (2004). Shape and the first hundred nouns. Child Development, 75, 1098-1114. http://dx.doi.org/10.1111/j.1467-8624.2004.00728.x

Hahn, E. R., \& Cantrell, L. (2012). The shape-bias in Spanish-speaking children and its relationship to vocabulary. Journal of Child Language, 39, 443-455. http://dx.doi.org/10.1017/S030500091100016X

Hatano, G., \& Osawa, K. (1983). Digit memory of grand experts in abacus-derived mental calculation. Cognition, 15(1-3), 95-110. http://dx.doi.org/10.1016/0010-0277(83)90035-5

Hershler, O., \& Hochstein, S. (2009). The importance of being expert: Top-down attentional control in visual search with photographs. Attention, Perception, \& Psychophysics, 71, 1478-1486. http://dx.doi.org/10.3758/APP.71.7.1478

Kornell, N., \& Bjork, R. A. (2008). Learning concepts and categories: Is spacing the "enemy of induction"? Psychological Science, 19(6), 585-592. http://dx.doi.org/10.1111/j.14679280.2008.02127.x

Landauer, T. K. (1969). Reinforcement as consolidation. Psychological Review, 76(1), 82-96. http://dx.doi.org/10.1037/h0026746

Lehmann, A. C., \& Ericsson, K. A. (1996). Performance without preparation: Structure and acquisition of expert sight-reading and accompanying performance. Psychomusicology: A Journal of Research in Music Cognition, 15(1-2), 1-29. http://dx.doi.org/10.1037/h0094082

Liang, K. Y., \& Zeger, S. L. (1986). Longitudinal data analysis using generalized linear models. Biometrika, 13-22. http://dx.doi.org/10.2307/2336267

Mack, A., \& Rock, I. (1998). Inattentional blindness. Cambridge, MA: MIT press. 
Mervis, C. B., Bertrand, J., \& Pani, J. R. (1995). Transaction of cognitive-linguistic abilities and adult input: A case study of the acquisition of colour terms and colour-based subordinate object categories. British Journal of Developmental Psychology, 13, 285-302. http://dx.doi.org/10.1111/j.2044-835X.1995.tb00680.x

Oliver, M., Schluter, P. J., \& Schofield, G. M. (2012). Body size, physical activity, and exposure to television in preschoolers. Open Journal of Preventive Medicine, 2, 359-363. http://dx.doi.org/10.4236/ojpm.2012.23052

Pan, W. (2001). Akaike's information criterion in generalized estimating equations. Biometrics, 57, 120-125. http://dx.doi.org/10.1111/j.0006-341X.2001.00120.x

Perry, L. K., Axelsson, E. L., \& Horst, J. S. (2015). Learning what to remember: Vocabulary knowledge and children's memory for object names and features. Infant and Child Development, 25, 247-258. http://dx.doi.org/10.1002/icd.1933

Perry, L. K., \& Saffran, J. R. (2016). Is a pink cow still a cow? Individual differences in toddlers' vocabulary knowledge and lexical representations. Cognitive Science, 1-16. http://dx.doi.org/10.1111/cogs. 12370

Perry, L. K., \& Samuelson, L. K. (2011). The shape of the vocabulary predicts the shape of the bias. Frontiers in Psychology, 2, 345. http://dx.doi.org/10.3389/fpsyg.2011.00345

Recht, D. R., \& Leslie, L. (1988). Effect of prior knowledge on good and poor readers' memory of text. Journal of Educational Psychology, 80(1), 16-20. http://dx.doi.org/10.1037/00220663.80.1.16

Samuelson, L. K., \& Smith, L. B. (1999). Early noun vocabularies: Do ontology, category structure and syntax correspond?. Cognition, 73(1), 1-33. http://dx.doi.org/10.1016/S0010-0277(99)00034-7 
Sandhofer, C. M., \& Smith, L. B. (1999). Learning color words involves learning a system of mappings. Developmental Psychology, 35, 668-679. http://dx.doi.org/10.1037/00121649.35.3.668

Shatz, M., Behrend, D., Gelman, S. A., \& Ebeling, K. S. (1996). Colour term knowledge in twoyear-olds: Evidence for early competence. Journal of Child Language, 23, 177-200. http://dx.doi.org/10.1017/S030500090001014X

Smith, L. B. (2000). Learning how to learn words: An associated crane. In R. M. Golinkoff, K. Hirsh-Pasek, L. Bloom, L. B. Smith, A. L. Woodward, N. Akhtar, M. Tomasello, \& G. Hollich (Eds.), Becoming a word learner: A debate on lexical acquisition (pp.51-80). New York: Oxford University Press.

Smith, L. B. (2001). How domain-general processes may create domain-specific biases. In M. Bowerman \& S. C. Levinson, Language acquisition and conceptual development (pp. 101-131). The Netherlands: Cambridge University Press.

Smith, L. B. (2005). Action alters shape categories. Cognitive Science, 29, 665-679. http://dx.doi.org/10.1207/s15516709cog0000_13

Smith, L. B., \& Samuelson, L. (2006). An attentional learning account of the shape bias: Reply to Cimpian and Markman (2005) and Booth, Waxman, and Huang (2005). Developmental Psychology, 42, 1339-1343. http://dx.doi.org/10.1037/00121649.42.6.1339

Vlach, H. A. (2014). The spacing effect in children's generalization of knowledge: Allowing children time to forget promotes their ability to learn. Child Development Perspectives, 8 , 163-168. http://dx.doi.org/10.1111/cdep.12079 
Vlach, H. A., Sandhofer, C. M., \& Kornell, N. (2008). The spacing effect in children's memory and category induction. Cognition, 109(1), 163-167. http://dx.doi.org/10.1016/j.cognition.2008.07.013

Vlach, H. A., Ankowski, A. A., \& Sandhofer, C. M. (2012). At the same time or apart in time? The role of presentation timing and retrieval dynamics in generalization. Journal of Experimental Psychology: Learning, Memory, and Cognition, 38, 246-254. http://dx.doi.org/10.1037/a0025260

Vlach, H. A., Sandhofer, C. M., \& Bjork, R. A. (2014). Equal spacing and expanding schedules in children's categorization and generalization. Journal of Experimental Child Psychology, 123, 129-137. http://dx.doi.org/10.1016/j.jecp.2014.01.004

Waxman, S. R., Senghas, A., \& Benveniste, S. (1997). A cross-linguistic examination of the noun-category bias: Its existence and specificity in French-and Spanish-speaking preschool-aged children. Cognitive Psychology, 32, 183-218. http://dx.doi.org/10.1006/cogp.1997.0650

Zeger, S. L., \& Liang, K. Y. (1986). Longitudinal data analysis for discrete and continuous outcomes. Biometrics, 42, 121-130. http://dx.doi.org/10.2307/2531248

Zucker, N., Copeland, W., Franz, L., Carpenter, K., Keeling, L., Angold, A., \& Egger, H. (2015). Psychological and psychosocial impairment in preschoolers with selective eating. Pediatrics, 136, e582-e590. http://dx.doi.org/10.1542/peds.2014-2386 